\title{
ON THE SYMMETRY BREAKING PHENOMENON
}

\author{
PETRE BIRTEA, MIRCEA PUTA, TUDOR S. RATIU AND RĂZVAN MICU TUDORAN
}

\begin{abstract}
We investigate the problem of symmetry breaking in the framework of dynamical systems with symmetry on a smooth manifold. Two cases will be analyzed: general and Hamiltonian dynamical systems. We give sufficient conditions for symmetry breaking in both cases.
\end{abstract}

\section{INTRODUCTION}

In this paper we investigate the problem of symmetry breaking in the framework of dynamical systems with symmetry on a smooth manifold. Two cases will be analyzed: general and Hamiltonian dynamical systems. For general vector fields we translate the problem locally to a symmetry breaking problem on a vector space where the techniques are more developed and easier to apply because of the existence of a Hilbert basis of invariant polynomials for representations of compact Lie groups. The symmetric steady state and Hopf bifurcations are considered as examples. Sufficient conditions for the existence of symmetry breaking bifurcations are known in this case. In the Hamiltonian context we find sufficient conditions for the existence of symmetry breaking bifurcations for Hamiltonian vector fields on symplectic vector spaces. The Hamiltonian steady state (passing of eigenvalues of the linearization on the imaginary axis at the origin) and the Hamiltonian Hopf bifurcations are analyzed. It turns out that the same methods as in the general case work, because in the Hamiltonian context one can work only with the invariant polynomials of the action and ignore the equivariant polynomials. In addition, it is shown how to locally translate these Hamiltonian bifurcation problems from the manifold to the tangent space at the bifurcation point. This is done by using the equivariant Darboux theorem.

\section{SOME BASIC RESUlts FROM THE THEORY OF GROUP ACTIONS}

We shall need a few fundamental results form the theory of group actions which we now briefly recall. For proofs and further information see [5], [7, [1], 20] and for the subsection on compact group representations see [12, [13, 23].

2.1. Twisted products. Let $G$ be a Lie group and $H \subset G$ a Lie subgroup. Supose that $H$ acts on the left on a manifold $A$. The twisted action of $H$ on the product $G \times A$ is defined by

$$
h \cdot(g, a)=\left(g h, h^{-1} \cdot a\right), \quad h \in H, \quad g \in G, \quad a \in A .
$$

Note that this action is free and proper by the freeness and properness of the action on the $G$-factor. The twisted product $G \times_{H} A$ is defined as the orbit space $(G \times A) / H$ of the twisted action. The elements of $G \times_{H} A$ will be denoted by $[g, a], g \in G, a \in A$. The twisted product $G \times_{H} A$ is a $G$-space relative to the left action defined by $g^{\prime} \cdot[g, a]=\left[g^{\prime} g, a\right]$. Also, the action of $H$ on $A$ is proper if and only if the $G$-action on $G \times{ }_{H} A$ is proper. The isotropy subgroups of the $G$-action on the twisted product $G \times{ }_{H} A$ satisfy

$$
G_{[g, a]}=g H_{a} g^{-1}, \quad g \in G, \quad a \in A .
$$


2.2. Slices. Throughout this paragraph it will be assumed that $\Psi: G \times Q \rightarrow Q$ is a left proper action of the Lie group $G$ on the manifold $Q$. This action will not be assumed to be free, in general. For $q \in Q$ we will denote by $H:=G_{q}:=\{g \in G \mid g \cdot q=q\}$ the isotropy subgroup of the action $\Psi$ at $q$. We shall introduce also the following convenient notation: if $K \subset G$ is a Lie subgroup of $G$ (possibly equal to $G), \mathfrak{k}$ its Lie algebra, and $q \in Q$, then $\mathfrak{k} \cdot q:=\left\{\eta_{Q}(q) \mid \eta \in \mathfrak{k}\right\}$ is the tangent space to the orbit $K \cdot q$ at $q$. A tube around the orbit $G \cdot q$ is a $G$-equivariant diffeomorphism $\varphi: G \times_{H} A \rightarrow U$, where $U$ is a $G$-invariant neighborhood of $G \cdot q$ and $A$ is some manifold on which $H$ acts. Note that the $G$-action on the twisted product $G \times{ }_{H} A$ is proper since the isotropy subgroup $H$ is compact and, consequently, its action on $A$ is proper. Hence the $G$-action on $G \times{ }_{H} A$ is proper. Let $S$ be a submanifold of $Q$ such that $q \in S$ and $H \cdot S=S$. We say that $S$ is a slice at $q$ if the map

$$
\varphi: G \times_{H} S \ni[g, s] \mapsto g \cdot s \in U
$$

is a tube about $G \cdot q$, for some $G$-invariant open neighborhood of $G \cdot q$. Notice that if $S$ is a slice at $q$ then $g \cdot S$ is a slice at the point $g \cdot q$. The following statements are equivalent:

(i) There is a tube $\varphi: G \times{ }_{H} A \rightarrow U$ about $G \cdot q$ such that $\varphi([e, A])=S$.

(ii) $S$ is a slice at $q$.

(iii) The submanifold $S$ satisfies the following properties:

(a) The set $G \cdot S$ is an open neighborhood of the orbit $G \cdot q$ and $S$ is closed in $G \cdot S$.

(b) For any $s \in S$ we have $T_{s} Q=\mathfrak{g} \cdot s+T_{s} S$. Moreover, $\mathfrak{g} \cdot s \cap T_{s} S=\mathfrak{h} \cdot s$, where $\mathfrak{h}$ is the Lie algebra of $H$. In particular $T_{q} Q=\mathfrak{g} \cdot q \oplus T_{q} S$.

(c) $S$ is $H$-invariant. Moreover, if $s \in S$ and $g \in G$ are such that $g \cdot s \in S$, then $g \in H$.

(d) Let $\sigma: U \subset G / H \rightarrow G$ be a local section of the submersion $G \rightarrow G / H$. Then the map $F: U \times S \rightarrow Q$ given by $F(u, s):=\sigma(u) \cdot s$ is a diffeomorphism onto an open set of $Q$.

(iv) $G \cdot S$ is an open neighborhood of $G \cdot q$ and there is an equivariant smooth retraction $r: G \cdot S \rightarrow G \cdot q$ of the injection $G \cdot q \hookrightarrow G \cdot S$ such that $r^{-1}(q)=S$.

Theorem 2.1. (Slice Theorem) Let $Q$ be a manifold and $G$ be a Lie group acting properly on $Q$ at the point $q \in Q$. Then there exists a slice for the $G$-action at $q$.

Theorem 2.2. (Tube Theorem) Let $Q$ be a manifold and $G$ a Lie group acting properly on $Q$ at the point $q \in Q, H:=G_{q}$. Then there is a tube $\varphi: G \times_{H} B \rightarrow U$ about $G \cdot q$ such that $\varphi([e, 0])=$ $q, \varphi([e, B])=: S$ is a slice at $q ; B$ is an open $H$-invariant neighborhood of 0 in the vector space $T_{q} Q / T_{q}(G \cdot q)$, on which $H$ acts linearly by $h \cdot\left(v_{q}+T_{q}(G \cdot q)\right):=T_{q} \Psi_{h}\left(v_{q}\right)+T_{q}(G \cdot q)$.

If $Q$ is a Riemannian manifold then $B$ can be chosen to be a $G_{q}$-invariant neighborhood of 0 in $(\mathfrak{g} \cdot q)^{\perp}$, the orthogonal complement to $\mathfrak{g} \cdot q$ in $T_{q} Q$. In this case $U=G \cdot \operatorname{Exp}_{q}(B)$, where $\operatorname{Exp}_{q}: T_{q} Q \rightarrow Q$ is the Riemannian exponential map.

2.3. Type submanifolds and fixed point subspaces. Let $G$ be a Lie group acting on a manifold $Q$. Let $H$ be a closed subgroup of $G$. We define the following subsets of $Q$ :

$$
\begin{aligned}
Q_{(H)} & =\left\{q \in Q \mid G_{q}=g H g^{-1}, g \in G\right\}, \\
Q^{H} & =\left\{q \in Q \mid H \subset G_{q}\right\}, \\
Q_{H} & =\left\{q \in Q \mid H=G_{q}\right\} .
\end{aligned}
$$

All these sets are submanifolds of $Q$. The set $Q_{(H)}$ is called the $(H)$-orbit type submanifold, $Q_{H}$ is the $H$-isotropy type submanifold, and $Q^{H}$ is the $H$-fixed point submanifold. We will collectively call these subsets the type submanifolds. We have:

- $Q^{H}$ is closed in $Q$; 
- $Q_{(H)}=G \cdot Q_{H}$;

- $Q_{H}$ is open in $Q^{H}$.

- the tangent space at $q \in Q_{H}$ to $Q_{H}$ equals

$$
T_{q} Q_{H}=\left\{v_{q} \in T_{q} Q \mid T_{q} \Psi_{h}\left(v_{q}\right)=v_{q} \text {, for all } h \in H\right\}=\left(T_{q} Q\right)^{H}=T_{q} Q^{H} ;
$$

- $T_{q}(G \cdot q) \cap\left(T_{q} Q\right)^{H}=T_{q}(N(H) \cdot q)$, where $N(H)$ is the normalizer of $H$ in $G$;

- if $H$ is compact then $Q_{H}=Q^{H} \cap Q_{(H)}$ and $Q_{H}$ is closed in $Q_{(H)}$.

If $Q$ is a vector space on which $H$ acts linearly, the set $Q^{H}$ is called in the physics literature the space of singlets or the space of invariant vectors.

Theorem 2.3. (The stratification theorm) Let $Q$ be a smooth manifold and $G$ be a Lie group acting properly on it. The connected components of the orbit type manifolds $Q_{(H)}$ and their projections onto the orbit space $Q_{(H)} / G$ constitute a Whitney stratification of $Q$ and $Q / G$, respectively. This stratification of $Q / G$ is minimal among all Whitney stratifications of $Q / G$.

The proof of this result, that can be found in [7] or [22, is based on the Slice Theorem and on a series of extremely important properties of the orbit type manifolds decomposition that we enumerate in what follows. We start by recalling that the set of conjugacy classes of subgroups of a Lie group $G$ admits a partial order by defining $(K) \preceq(H)$ if and only if $H$ is conjugate to a subgroup of $K$. Also, a point $q \in Q$ in a proper $G$-subspace of $Q$ (or its corresponding $G$-orbit, $G \cdot q$ ) is called principal if its corresponding local orbit type manifold is open in $Q$. The orbit $G \cdot q$ is called regular if the dimension of the orbits nearby coincides with the dimension of $G \cdot q$. The set of principal and regular orbits will be denoted by $Q_{\text {princ }} / G$ and $Q_{r e g} / G$, respectively. Using this notation we have:

- For any $q \in Q$ there exists a neighborhood $U$ of $q$ that intersects only finitely many connected components of finitely many orbit type manifolds. If $Q$ is compact or a linear space where $G$ acts linearly, then the $G$-action on $Q$ has only finitely many distinct connected components of orbit type manifolds.

- For any $q \in Q$ there exists an open neigborhood $U$ of $q$ such that $\left(G_{q}\right) \preceq\left(G_{x}\right)$, for all $x \in U$. In particular, this implies that $\operatorname{dim} G \cdot q \leq \operatorname{dim} G \cdot x$, for all $x \in U$.

- Principal Orbit Theorem: For every connected component $Q^{0}$ of $Q$ the subset $Q_{\text {princ }} \cap Q^{0}$ is connected, open, and dense in $Q^{0}$. Each connected component $(Q / G)^{0}$ of $Q / G$ contains only one principal orbit type, which is connected open and dense in $(Q / G)^{0}$.

2.4. General facts about compact Lie groups representations. Let $G$ be a compact Lie group acting on a finite dimensional vector space $V$. The compactness of $G$ implies the existence of a $G$ invariant inner product $\langle\langle\cdot, \cdot\rangle\rangle$ on $V$, so one can always assume that the $G$-representation is orthogonal. Denote by $\mathcal{P}_{G}(V)$ the ring of $G$-invariant real valued polynomials on $V$. The Hilbert-Weyl theorem implies that $\mathcal{P}_{G}(V)$ is finitely generated over $\mathbb{R}$ and it can be shown that one can choose the generators to be homogeneous polynomials. The elements $\left\{\theta_{1}, \ldots, \theta_{l}\right\}$ of such a basis over $\mathbb{R}$ are called invariant generators. The following theorem of Schwarz gives a method of writing a $G$-equivariant smooth map in terms of invariants and equivariants.

Theorem 2.4. Let $V$ and $W$ be two $G$-representation spaces. The set of $G$-equivariant polynomial maps from $V$ to $W$ is a finitely generated $\mathcal{P}_{G}(V)$-module. Let $\left\{F_{1}, \ldots, F_{k}\right\}$ be one of its minimal bases. Then, if $F$ is a smooth $G$-equivariant map from $V$ to $W$, there exist smooth functions $f_{1}, \ldots, f_{k}$ on $\mathbb{R}^{l}$ such that

$$
F(v)=\sum_{i=1}^{k} f_{i}\left(\theta_{1}(v), \ldots, \theta_{l}(v)\right) F_{i}(v) .
$$


The next theorem gives an identification between the orbit space of a linear action of a compact Lie group $G$ on a vector space $V$ and a semialgebraic subset of $\mathbb{R}^{l}$.

Theorem 2.5. Let $\pi$ be the Hilbert map $V \ni v \mapsto\left(\theta_{1}(v), \ldots, \theta_{l}(v)\right) \in \mathbb{R}^{l}$. Then $\pi$ induces a homeomorphism between the orbit space $V / G$ and $\operatorname{Im}(\pi)$.

The invariant generators are intimately related to the orthogonal space to the $G$-orbits. If $v \in V$, let $N_{v}:=\{u \in V|\langle u, \xi \cdot v\rangle\rangle=0$, for all $\xi \in \mathfrak{g}\}$ be the orthogonal space to the orbit $G \cdot v$. Define the normal invariant subspace $N_{v}^{(0)}:=\left\{u \in N_{v} \mid g \cdot u=u\right.$, for all $\left.g \in G_{v}\right\} \subset N_{v}$. The following theorem is proved in 3 .

Theorem 2.6. The vector subspace of $V$ generated by $\left\{\operatorname{grad} \theta_{i}(v) \mid i=1, \ldots, l\right\}$ coincides with the normal invariant subspace $N_{v}^{(0)}$.

Two useful consequences of this fact are the orthogonal sum decomposition

$$
T_{v} V_{(H)}=T_{v}(G \cdot v) \oplus N_{v}^{(0)}
$$

and the fact that $F_{i}(v) \in T_{v}\left(V_{\left(G_{v}\right)}\right)$ for any equivariant generator $F_{i}, i=1, \ldots, k$ of the $G$-representation. Since the orbit space is the image of a finite dimensional vector space by a polynomial map, the Tarski-

Seidenberg theorem guarantees that it is a semialgebraic set. Thus, as a semialgebraic set, $V / G$ has a canonical stratification. A theorem of Bierstone implies that this canonical semialgebraic stratification is the same as the stratification by orbit types obtained by projection of those from $V$. Since the orbit space $V / G$ inherits a smooth structure from $V$ and $G$, but in general is not a manifold, we have to define the meaning of a smooth vector field on $V / G$.

Definition 2.7. Let $X$ be a smooth vector field defined on a neighborhood $U$ of $0 \in \mathbb{R}^{l}$ and tangent to the strata of $\pi(V) \cap U$. Then $X$ is said to be smooth vector field on $V / G$ in a neighborhood of the origin.

If one projects a smooth $G$-equivariant vector field on the orbit space, one obtains a smooth vector field on $V / G$ in the sense defined above. When we speak of a vector field on $V / G$ we mean a vector field which is tangent to each stratum of $V / G$. The next step gives a method for projecting a $G$-equivariant vector field on the orbit space. For doing this, let $F$ be a smooth $G$-equivariant vector field on the finite dimensional vector space $V$. Let $\left\{\theta_{1}, \ldots, \theta_{l}\right\}$ be a set of generators for $\mathcal{P}_{G}(V)$ and let $\left\{F_{1}, \ldots, F_{k}\right\}$ be a set of generators for the $\mathcal{P}_{G}(V)$-module of $G$-equivariant polynomials maps from $V$ to $V$. By the Schwarz theorem we can write $F$ in terms of invariants and equivariants as

$$
F(v)=\sum_{i=1}^{k} f_{i}\left(\theta_{1}(v), \ldots, \theta_{l}(v)\right) F_{i}(v),
$$

where the functions $f_{i}$ are smooth functions on $\mathbb{R}^{l}$. To obtain the projection of this vector field on the orbit space $V / G$, one has to compute the derivatives of the invariant generators $\theta_{i}$ with respect to time:

$$
\begin{aligned}
\dot{\theta}_{j} & =\left\langle\left\langle\operatorname{grad}_{v}\left(\theta_{j}\right), \dot{v}\right\rangle\right\rangle=\left\langle\left\langle\operatorname{grad}_{v}\left(\theta_{j}\right), \sum_{i=1}^{k} f_{i}(\theta(v)) F_{i}(v)\right\rangle\right\rangle \\
& =\sum_{i=1}^{k} f_{i}(\theta(v))\left\langle\left\langle\operatorname{grad}_{v}\left(\theta_{j}\right), F_{i}(v)\right\rangle\right\rangle .
\end{aligned}
$$


Since the expression $\left\langle\left\langle\operatorname{grad}_{v}\left(\theta_{j}\right), F_{i}(v)\right\rangle\right\rangle$ is $G$-invariant one can rewrite it in terms of the invariant generators. Thus the projection $\widetilde{F}$ of $F$ on the orbit space $V / G$ has the expression

$$
\widetilde{F}(\theta)=\sum_{i=1}^{k} f_{i}(\theta) \widetilde{F}_{i}(\theta),
$$

where if $v$ is such that $\pi(v)=\theta$ and

$$
\widetilde{F}_{i}(\theta):=\left[\begin{array}{c}
\left\langle\left\langle F_{i}(v), \operatorname{grad}_{v} \theta_{1}(v)\right\rangle\right\rangle \\
\vdots \\
\left\langle\left\langle F_{i}(v), \operatorname{grad}_{v} \theta_{l}(v)\right\rangle\right\rangle
\end{array}\right] \quad \text { for } \quad i=1, \ldots, k .
$$

\section{Formulation of Symmetric Systems on Vector Spaces}

This section presents a method of translating a bifurcation problem for a general vector fields family with $G$-symmetry from a smooth manifold to a vector space. We begin by quickly recalling the necessary notations and statements for a left smooth proper Lie group action $\Phi: G \times Q \rightarrow Q$, where $Q$ is a smooth manifold and $G$ is a Lie group with Lie algebra $\mathfrak{g}$.

Definition 3.1. A vector field $X: Q \rightarrow T Q$ is said to be $G$-equivariant if

$$
T_{q} \Phi_{g}(X(q))=X\left(\Phi_{g}(q)\right)
$$

for all $q \in Q$ and $g \in G$. If $X$ is $G$-equivariant, then $G$ is said to be a symmetry group of the dynamical system $\dot{q}=X(q)$.

Definition 3.2. A relative equilibrium of a $G$-equivariant vector field $X$ is a point $q_{e} \in Q$ at which the values of $X$ and of the infinitesimal generator $\xi_{Q}$ of some element $\xi \in \mathfrak{g}$, called velocity of the relative equilibrium, coincide, that is,

$$
X\left(q_{e}\right)=\xi_{Q}\left(q_{e}\right):=\left.\frac{d}{d t}\right|_{t=0} \Phi\left(\exp t \xi, q_{e}\right),
$$

where $\exp : \mathfrak{g} \rightarrow G$ is the Lie group exponential map. A relative equilibrium $q_{e}$ is said to be asymmetric if $\mathfrak{g}_{q_{e}}=\{0\}$, and symmetric otherwise.

From the definition above and the uniqness of the integral curves of a vector field with given initial condition, it follows that the dynamical orbit $q(t)$ of $X$ staring at the relative equilibrium $q_{e}$, that is, $q(0)=q_{e}$, coincides with the curve $\exp t \xi \cdot q_{e}:=\Phi\left(\exp t \xi, q_{e}\right)$ given by the group action. Note that if $\xi$ is a velocity of the relative equilibrium $q_{e}$, then so is $\xi+\eta$, where $\eta \in \mathfrak{g}_{q_{e}}:=\left\{\zeta \in \mathfrak{g} \mid \zeta_{Q}\left(q_{e}\right)=0_{q_{e}}\right\}$. The Lie subalgebra $\mathfrak{g}_{q_{e}}$ is called the symmetry algebra of $q_{e}$. Denote for each $g \in G$ by Ad $: G \times \mathfrak{g} \rightarrow \mathfrak{g}$ the adjoint representation of $G$ on $\mathfrak{g}$; for each $g \in G, \operatorname{Ad}_{g}$ is the Lie algebra isomorphism obtained by taking the derivative of the conjugation isomorphism on $G$.

Proposition 3.3. If $q_{e}$ is a relative equilibrium with velocity $\xi \in \mathfrak{g}$, then, for any $g \in G, \Phi_{g}\left(q_{e}\right)$ is also a relative equilibrium with velocity $\operatorname{Ad}_{g} \xi$.

Proof. Indeed, since $X$ is $G$-equivariant, we get

$$
X\left(\Phi_{g}\left(q_{e}\right)\right)=T_{q_{e}} \Phi_{g}\left(X\left(q_{e}\right)\right)=\left(T_{q_{e}} \Phi_{g} \circ \xi_{Q} \circ \Phi_{g^{-1}}\right)\left(\Phi_{g}\left(q_{e}\right)\right)=\left(\operatorname{Ad}_{g} \xi\right)_{Q}\left(\Phi_{g}\left(q_{e}\right)\right),
$$

which proves the statement.

By one of the remarks following Theorem 2.3 we can choose a $G$-invariant tubular open neigborhood $W_{q_{e}}$ of the orbit $G \cdot q_{e}$ such that the isotropy subgroup $G_{x}$ for any $x \in W_{q_{e}}$ is conjugate to a (possibly non-strict) subgroup of $G_{q_{e}}$. The next lemma will show that we can do better. 
Lemma 3.4. Let $L$ be a compact Lie group acting on a smooth manifold $Q$. Assume that $q \in Q$ is a fixed point of the L-action. Then any open neighborhood of $q$ contains a L-invariant open neighborhood of $q$.

Proof. Let $\Phi: L \times Q \rightarrow Q$ be the group action and $U$ an arbitrary neighborhood of $q$. The set $\Phi^{-1}(U)$ is clearly open and contains $L \times\{q\}$. By continuity of $\Phi$, for any $g \in L$, there are open neighborhoods $W_{g}$ of $g$ in $L$ and $V_{g}$ of $q$ in $Q$ such that $W_{g} \times V_{g} \subset \Phi^{-1}(U)$. Since, by hypothesis, $L$ is compact, the open cover $\left\{W_{g} \mid g \in L\right\}$ of $L$ has a finite subcover $\left\{W_{g_{1}}, \ldots, W_{g_{n}}\right\}$. Let $V:=\bigcap_{i=1}^{n} V_{g_{i}}$. The $L$-invariant set $W:=\Phi(L, V) \subset U$ is clearly open since $W=\bigcup_{g \in L} \Phi_{g}(V)$ and, by construction, contains the point $q$.

Properness of the $G$-action on $Q$ implies that the isotropy subgroup $G_{q_{e}}$ is compact. By the lemma above we can conclude that there exists an open $G_{q_{e}}$-invariant neighborhood $W_{q_{e}}^{1}$ of $q_{e}$ contained in $W_{q_{e}}$. Thus the local study of the symmetry breaking from $G_{q_{e}}$ to a smaller symmetry group can be done by restricting the action to $W_{q_{e}}^{1}$. Next, assume that $Q$ carries a $G$-invariant Riemannian metric. The Riemannian exponential map $\operatorname{Exp}: T Q \rightarrow Q$ is also $G$-equivariant, relative to the naturally lifted $G$-action on $T Q$. Since $\operatorname{Exp}_{q_{e}}: T_{q_{e}} Q \rightarrow Q$ is a local diffeomorphism around the origin, we can further shrink the neighborhood $W_{q_{e}}^{1}$ (keeping the $G_{q_{e}}$-invariance), till it is contained in the image under $\operatorname{Exp}_{q_{e}}$ of the neighborhood of the origin where $\operatorname{Exp}_{q_{e}}$ is a diffeomorphism. Denote this shrunk $G_{q_{e}}$-invariant neighborhood by $U_{q_{e}}$. The inverse map of the Riemannian exponential on $U_{q_{e}}, \log _{q_{e}}$ : $U_{q_{e}} \rightarrow \log _{q_{e}}\left(U_{q_{e}}\right) \subset T_{q_{e}} Q$ is therefore also $G_{q_{e}}$-equivariant and so its image $\log _{q_{e}}\left(U_{q_{e}}\right)=: V_{0_{q_{e}}}$ will be a $G_{q_{e}}$-invariant open neighborhood of $0_{q_{e}} \in T_{q_{e}} Q$.

Proposition 3.5. Let $(Q,\langle\langle\cdot, \cdot\rangle\rangle)$ be a smooth Riemannian manifold, $q_{e} \in Q, G$ a Lie group acting properly and smoothly on $Q$ by isometries, and $X \in \mathfrak{X}(Q)$ a smooth $G$-equivariant vector field. Then, in the notations above, the vector field $\operatorname{Exp}_{q_{e}}^{*}\left(\left.X\right|_{U_{q_{e}}}\right) \in \mathfrak{X}\left(V_{0_{q_{e}}}\right)$ is a smooth $G_{q_{e}}$-equivariant vector field on the open neighborhood $V_{0_{q_{e}}}$ of the origin $0_{q_{e}}$ in the vector space $T_{q_{e}} Q$.

Proof. The proof is a simple consequence of the fact that $\operatorname{Exp}_{q_{e}}: V_{q_{e}} \subset T_{q_{e}} Q \rightarrow U_{q_{e}} \subset Q$ and $\log _{q_{e}}: U_{q_{e}} \rightarrow V_{q_{e}}$ are $G_{q_{e}}$-equivariant diffeomorphisms inverse to each other. Indeed, since $X$ is $G$ equivariant, its restriction $\left.X\right|_{U_{q_{e}}}$ is $G_{q_{e}}$-equivariant. Therefore, for any $h \in G_{q_{e}}$ and $v_{q_{e}} \in V_{q_{e}}$ we have

$$
\begin{aligned}
\left(\operatorname{Exp}_{q_{e}}^{*}\left(\left.X\right|_{U_{q_{e}}}\right)\right)\left(T_{q_{e}} \Phi_{h}\left(v_{q_{e}}\right)\right) & \\
& =T_{\operatorname{Exp}_{q_{e}}\left(T_{q_{e}} \Phi_{h}\left(v_{q_{e}}\right)\right)} \log _{q_{e}}\left(X\left(\operatorname{Exp}_{q_{e}}\left(T_{q_{e}} \Phi_{h}\left(v_{q_{e}}\right)\right)\right)\right) \\
& =T_{\Phi_{h}\left(\operatorname{Exp}_{q_{e}}\left(v_{q_{e}}\right)\right)} \log _{q_{e}}\left(X\left(\Phi_{h}\left(\operatorname{Exp}_{q_{e}}\left(v_{q_{e}}\right)\right)\right)\right) \\
& =T_{\operatorname{Exp}_{q_{e}}\left(v_{q_{e}}\right)}\left(\log _{q_{e}} \circ \Phi_{h}\right)\left(X\left(\operatorname{Exp}_{q_{e}}\left(v_{q_{e}}\right)\right)\right) \\
& =T_{\operatorname{Exp}_{q_{e}}\left(v_{q_{e}}\right)}\left(T_{q_{e}} \Phi_{h} \circ \log _{q_{e}}\right)\left(X\left(\operatorname{Exp}_{q_{e}}\left(v_{q_{e}}\right)\right)\right) \\
& =T_{v_{q_{e}}}\left(T_{q_{e}} \Phi_{h}\right)\left(\left(\operatorname{Exp}_{q_{e}}^{*}\left(\left.X\right|_{U_{q_{e}}}\right)\right)\left(v_{q_{e}}\right)\right)
\end{aligned}
$$

which proves that $\operatorname{Exp}_{q_{e}}^{*}\left(\left.X\right|_{U_{q_{e}}}\right)$ is a $G_{q_{e}}$-equivariant vector field on $V_{0_{q_{e}}}$.

Proposition 3.6. Let $(Q,\langle\langle\cdot, \cdot\rangle\rangle)$ be a smooth Riemannian manifold, $G$ a Lie group acting properly on $Q$ by isometries, and $X_{\lambda} \in \mathfrak{X}(Q), \lambda \in \mathbb{R}$, a family of smooth $G$-equivariant vector fields. Suppose that $q_{e} \in Q$ is a (relative) equilibrium for $X_{\lambda_{0}}$ with symmetry $G_{q_{e}}$. Assume the family of vector fields $\operatorname{Exp}_{q_{e}}^{*} X_{\lambda} \in \mathfrak{X}\left(V_{0_{q_{e}}}\right)$ has the property that there exist $\epsilon>0$ and a continuous curve $\gamma:\left[\lambda_{0}, \lambda_{0}+\epsilon\right] \rightarrow V_{0_{q_{e}}}$, $\gamma\left(\lambda_{0}\right)=0_{q_{e}}$, such that all the points on the curve $\gamma(\lambda)$ for $\lambda>\lambda_{0}$ are relative equilibria of $\operatorname{Exp}_{q_{e}}^{*} X_{\lambda}$ with isotropy subgroups conjugate to $H \subset G_{q_{e}}$. Then the curve $\operatorname{Exp}_{q_{e}}$ o $\gamma:\left[\lambda_{0}, \lambda_{0}+\epsilon\right] \rightarrow U_{q_{e}}$ is formed by relative equilibria of $X_{\lambda}$ with isotropy subgroups conjugate to $H$ for all $\lambda>\lambda_{0}$ and to $G_{q_{e}}$ for $\lambda=\lambda_{0}$. 
Proof. Let $X_{\lambda} \in \mathfrak{X}\left(U_{q_{e}}\right)$ be one of the vector fields restricted to the $G_{q_{e}}$-invariant open neighborhood $U_{q_{e}}$. Suppose that $\gamma(\lambda)$ is a relative equilibrium of the vector field $\operatorname{Exp}_{q_{e}}^{*} X_{\lambda}$. This means that there exists an element $\xi(\lambda) \in \mathfrak{g}_{q_{e}} \subset \mathfrak{g}$ such that

$$
\left(\operatorname{Exp}_{q_{e}}^{*} X_{\lambda}\right)(\gamma(\lambda))=(\xi(\lambda))_{T_{q_{e}} Q}(\gamma(\lambda)),
$$

that is,

$$
T_{\operatorname{Exp}_{q_{e}}(\gamma(\lambda))} \log _{q_{e}}\left(X_{\lambda}\left(\operatorname{Exp}_{q_{e}}(\gamma(\lambda))\right)\right)=(\xi(\lambda))_{T_{q_{e}} Q}(\gamma(\lambda)) .
$$

If we apply to the above equality $T_{\gamma(\lambda)} \operatorname{Exp}_{q_{e}}$ and use the $G_{q_{e}}$-equivariance of the exponential map we get

$$
\begin{aligned}
X_{\lambda}\left(\operatorname{Exp}_{q_{e}}(\gamma(\lambda))\right) & =T_{\gamma(\lambda)} \operatorname{Exp}_{q_{e}}\left((\xi(\lambda))_{T_{q_{e}} Q}(\gamma(\lambda))\right) \\
& =(\xi(\lambda))_{Q}\left(\operatorname{Exp}_{q_{e}}(\gamma(\lambda))\right) .
\end{aligned}
$$

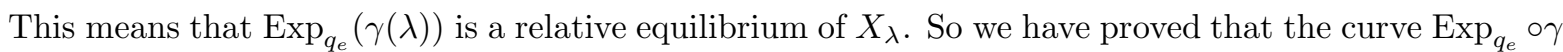
is formed by relative equilibria of $X_{\lambda}$ for any $\lambda \in\left[\lambda_{0}, \lambda_{0}+\epsilon\right]$. Because the exponential map is a $G_{q_{e}}{ }^{-}$ equivariant diffeomorphism on the neighborhoods considered, we have $G_{\operatorname{Exp}_{q_{e}}(\gamma(\lambda))}=G_{\gamma(\lambda)}$, and so all the points on the curve $\gamma(\lambda)$ with the possible exception of $\gamma\left(\lambda_{0}\right)$ are conjugate to $H$.

Since the $G_{q_{e}}$-equivariant family of vector fields $\operatorname{Exp}_{q_{e}}^{*} X_{\lambda} \in \mathfrak{X}\left(V_{0_{q_{e}}}\right)$ are defined on an open $G_{q_{e}}{ }^{-}$ invariant neighborhood of the origin in the vector space $T_{q_{e}} Q$, we can arbitrarily extend their restrictions to a smaller $G_{q_{e}}$-invariant neighborhood of the origin to smooth $G_{q_{e}}$-equivariant vector fields $\overline{X_{\lambda}} \in$ $\mathfrak{X}\left(T_{q_{e}} Q\right)$ on the whole space $T_{q_{e}} Q$. However, on $T_{q_{e}} Q$ we have an orthogonal representation of the compact group $G_{q_{e}}$ and thus, as explained earlier, the vector fields $\overline{X_{\lambda}} \in \mathfrak{X}\left(T_{q_{e}} Q\right)$ can be expressed in terms of the invariant and equivariant polynomials on $T_{q_{e}} Q$. Thus to study the local symmetry breaking phenomenon around the point $q_{e} \in Q$, one can translate the problem to the vector space $T_{q_{e}} Q$ and study this problem for the $G_{q_{e}}$-equivariant family $\overline{X_{\lambda}} \in \mathfrak{X}\left(T_{q_{e}} Q\right)$.

\section{Stationary and Hopf Bifurcation with Symmetry}

We shall analyze now the local symmetry breaking phenomenon around $0_{q_{e}} \in T_{q_{e}} Q$ for the family of $G_{q_{e}}$-equivariant vector fields $\overline{X_{\lambda}} \in \mathfrak{X}\left(T_{q_{e}} Q\right)$ under the following two nondegeneracy conditions:

- Stationary bifurcation with symmetry:

$$
D \overline{X_{\lambda}}\left(0_{q_{e}}\right)=\sigma_{X}(\lambda) \operatorname{Id}_{T_{q_{e}} Q}
$$

where $\sigma_{X}:(-\epsilon, \epsilon) \rightarrow \mathbb{R}$ is a smooth map satisfying $\sigma_{X}(0)=0$ and $\sigma_{X}^{\prime}(0) \neq 0$. In addition, it is assumed that the compact group $G_{q_{e}}$ acts absolutely irreducibly on $T_{q_{e}} Q$, i.e., that only scalar multiples of the identity commute with the $G_{q_{e}}$-action.

- Hopf bifurcation with symmetry:

$$
D \overline{X_{\lambda}}\left(0_{q_{e}}\right)=\sigma_{X}(\lambda) \operatorname{Id}_{T_{q_{e} Q}}+\rho_{X}(\lambda) \mathbb{d}_{T_{q_{e}} Q}
$$

where $\sigma_{X}, \rho_{X}:(-\epsilon, \epsilon) \rightarrow \mathbb{R}$ are smooth maps satisfying $\sigma_{X}(0)=0, \sigma_{X}^{\prime}(0) \neq 0$, and $\rho_{X}(0) \neq 0$. In addition, it is assumed that the vector space $T_{q_{e}} Q$ carries a natural complex structure $\mathbb{J}_{T_{q_{e}} Q}$ and that the compact group $G_{q_{e}}$ acts on this complex vector space $T_{q_{e}} Q$ irreducibly (as a complex representation). In this case the manifold $Q$ is necessarily of even dimension.

In the following will denote by $\mathcal{C}^{\infty}\left(T_{q_{e}} Q \times \mathbb{R}, T_{q_{e}} Q\right)_{o}^{G_{q_{e}}}$ the class of $G_{q_{e}}$-equivariant vector fields that verifies one of the above nondegeneracy conditions. 
Let $\left\{\theta_{1}\left(v_{q_{e}}\right), \ldots, \theta_{l}\left(v_{q_{e}}\right)\right\}$ be a set of generators for $\mathcal{P}_{G_{q_{e}}}\left(T_{q_{e}} Q\right)$ and let $\left\{F_{1}, \ldots, F_{k}\right\}$ be a set of generators for the $\mathcal{P}_{G_{q_{e}}}\left(T_{q_{e}} Q\right)$-module of $G_{q_{e}}$-equivariant maps from $T_{q_{e}} Q$ to itself. Then by the Schwarz theorem we have

$$
\overline{X_{\lambda}}\left(v_{q_{e}}\right)=\sum_{j=1}^{k} f_{j}\left(\theta_{1}\left(v_{q_{e}}\right), \ldots, \theta_{l}\left(v_{q_{e}}\right), \lambda\right) F_{j}\left(v_{q_{e}}\right),
$$

where the functions $f_{i}$ are smooth functions on $\mathbb{R}^{l}$. The projection of this family of vector fields on the orbit space $\left(T_{q_{e}} Q\right) / G_{q_{e}}$ has the expression:

$$
\dot{\theta}=\sum_{i=1}^{k} f_{i}(\theta, \lambda) \widetilde{F}_{i}(\theta)
$$

where, if $v_{q_{e}} \in T_{q_{e}} Q$ is such that $\pi\left(v_{q_{e}}\right)=\theta \in\left(T_{q_{e}} Q\right) / G_{q_{e}}$, we have

$$
\widetilde{F}_{i}(\theta)=\left[\begin{array}{c}
\left\langle\left\langle F_{i}\left(v_{q_{e}}\right), \operatorname{grad}_{v_{q_{e}}} \theta_{1}\left(v_{q_{e}}\right)\right\rangle\right\rangle \\
\vdots \\
\left\langle\left\langle F_{i}\left(v_{q_{e}}\right), \operatorname{grad}_{v_{q_{e}}} \theta_{l}\left(v_{q_{e}}\right)\right\rangle\right.
\end{array}\right] \quad \text { for } \quad i=1, \ldots, k .
$$

According to the orbit space projection of the vector field $\overline{X_{\lambda}}$ we introduce the maps

$$
\begin{gathered}
\widetilde{F}:\left(T_{q_{e}} Q\right) / G_{q_{e}} \times \mathbb{R}^{k} \ni(\theta, t) \mapsto \sum_{i=1}^{k} t_{i} \widetilde{F}_{i}(\theta) \in\left(T_{q_{e}} Q\right) / G_{q_{e}} \\
\gamma: \mathbb{R} \ni \lambda \mapsto\left(f_{1}(0, \lambda), \ldots, f_{k}(0, \lambda)\right) \in \mathbb{R}^{k}
\end{gathered}
$$

and define the sets:

$$
\begin{gathered}
\widetilde{\mathcal{E}}=\left\{(\theta, t) \in\left(T_{q_{e}} Q\right) / G_{q_{e}} \times \mathbb{R}^{k} \mid \widetilde{F}(\theta, t)=0\right\} \\
\widetilde{\mathcal{E}}_{(H)}=\left\{(\theta, t) \in \widetilde{\mathcal{E}} \mid \theta \in \Pi\left(\left(T_{q_{e}} Q\right)_{(H)}\right)\right\}, \\
\widetilde{\mathcal{A}}_{(H)}=\overline{\widetilde{\mathcal{E}}_{(H)}} \cap\left(\{[0]\} \times \mathbb{R}^{k}\right),
\end{gathered}
$$

where $\Pi: T_{q_{e}} Q \rightarrow\left(T_{q_{e}} Q\right) / G_{q_{e}}$ is the orbit space projection and $H \subset G_{q_{e}}$ is an isotropy subgroup of the $G_{q_{e}}$-action on $T_{q_{e}} Q$. Recall that $\widetilde{\mathcal{E}}$ is a semialgebraic subset of $\mathbb{R}^{l} \times \mathbb{R}^{k}$. Denote by $\Sigma$ its canonical Whitney stratification. This stratification induces a Whitney semialgebraic stratification $\Sigma_{(H)}$ on each $\widetilde{\mathcal{E}}_{(H)}$. Let $\mathcal{B}$ denote the Whitney stratification $\Sigma_{\left(G_{q_{e}}\right)}$ of $\widetilde{\mathcal{E}}_{\left(G_{q_{e}}\right)}=\{0\} \times \mathbb{R}^{k}$. Next we will define a notion of $G_{q_{e}}$-transversality introduced by Kœnig and Chossat [13] that was inspired by Bierstone [4] and Field 8 .

Definition 4.1. The projection to the orbit space $\left(T_{q_{e}} Q\right) / G_{q_{e}}$ of a $G_{q_{e}}$-equivariant smooth family of vector fields $\overline{X_{\lambda}} \in \mathfrak{X}\left(T_{q_{e}} Q\right)$ is transverse to $\left[0_{q_{e}}\right] \in\left(T_{q_{e}} Q\right) / G_{q_{e}}$ at $\lambda=0 \in \mathbb{R}$ if $\gamma: \mathbb{R} \rightarrow \mathbb{R}^{k}$ is transverse at $\lambda=0$ to the stratum of $\mathcal{B}$ containing $\gamma(0)$, that is, im $T_{0} \gamma+T_{\gamma(0)} S=\mathbb{R}^{k}$, where $S$ is the stratum of $\mathcal{B}$ containing $\gamma(0)$.

Analogous to the map (4.3) we define

$$
F: T_{q_{e}} Q \times \mathbb{R}^{k} \ni\left(v_{q_{e}}, t\right) \mapsto \sum_{i=1}^{k} t_{i} F_{i}\left(v_{q_{e}}\right) \in T_{q_{e}} Q .
$$

This map induces the following sets parameterized by the isotropy type subgroups of the $G_{q_{e}}$-action on $T_{q_{e}} Q$ :

$$
\mathcal{E}_{(H)}=\left\{\left(v_{q_{e}}, t\right) \in\left(T_{q_{e}} Q\right)_{(H)} \times \mathbb{R}^{k} \mid F\left(v_{q_{e}}, t\right) \in \mathfrak{g}_{q_{e}} \cdot v_{q_{e}}\right\}
$$




$$
\mathcal{A}_{(H)}=\overline{\mathcal{E}_{(H)}} \cap\left(\{0\} \times \mathbb{R}^{k}\right) .
$$

The sets $\mathcal{E}_{(H)}$ and $\mathcal{A}_{(H)}$ are semialgebraic sets in $T_{q_{e}} Q \times \mathbb{R}^{k}$ and $\mathbb{R}^{k}$ respectively. The next proposition gives an relation between the pair of subsets $\widetilde{\mathcal{E}}_{(H)} \subset\left(T_{q_{e}} Q\right) / G_{q_{e}} \times \mathbb{R}^{k}, \widetilde{\mathcal{A}}_{(H)} \subset \mathbb{R}^{k}$ and $\mathcal{E}_{(H)} \subset\left(T_{q_{e}} Q\right)_{(H)} \times$ $\mathbb{R}^{k}, \mathcal{A}_{(H)} \subset \mathbb{R}^{k}$.

Proposition 4.2. Let $\Pi: T_{q_{e}} Q \rightarrow\left(T_{q_{e}} Q\right) / G_{q_{e}}$ be the projection onto the orbit space and $H$ and isotropy type of the $G_{q_{e}}$-action on $T_{q_{e}} Q$. Then:

$$
\begin{gathered}
\mathcal{E}_{(H)}=(\Pi \times \mathrm{id})^{-1}\left(\widetilde{\mathcal{E}}_{(H)}\right), \\
\mathcal{A}_{(H)}=\widetilde{\mathcal{A}}_{(H)} .
\end{gathered}
$$

Proof. The proof is as in [12. To show the first equality let $v_{q_{e}} \in \Pi^{-1}(\theta)$. Then

$$
\widetilde{F}(\theta, t)=0 \Leftrightarrow\left\langle\left\langle F\left(v_{q_{e}}, t\right), \operatorname{grad}_{v_{q_{e}}} \theta_{j}\left(v_{q_{e}}\right)\right\rangle\right\rangle=0,
$$

for any $j \in\{1, \ldots, l\}$. As $F\left(v_{q_{e}}, t\right) \in T_{v_{q_{e}}}\left(T_{q_{e}} Q\right)_{\left(G_{v_{e}}\right)}=\mathfrak{g}_{q_{e}} \cdot v_{q_{e}} \oplus N_{v_{q_{e}}}^{(0)}$ and $N_{v_{q_{e}}}^{(0)}$ is generated by $\left\{\operatorname{grad}_{v_{q_{e}}} \theta_{j}\left(v_{q_{e}}\right)\right.$, for $\left.j \in\{1, \ldots, l\}\right\}$, we must have $F\left(v_{q_{e}}, t\right) \in \mathfrak{g}_{q_{e}} \cdot v_{q_{e}}$. Thus $(\theta, t) \in \widetilde{\mathcal{E}}_{(H)} \Longleftrightarrow\left(v_{q_{e}}, t\right) \in$ $\mathcal{E}_{(H)}$. To prove the second equality let $t \in \mathcal{A}_{(H)}$. Then there exists $\left(v_{n}, t_{n}\right) \in \mathcal{E}_{(H)}$ such that $v_{n} \rightarrow 0$ and $t_{n} \rightarrow t$. By the first equality, this implies that $\left(\Pi\left(v_{n}\right), t_{n}\right) \in \widetilde{\mathcal{E}}_{(H)}$ and the continuity of $\Pi$ implies that $t \in \widetilde{\mathcal{A}}_{(H)}$. Conversely, let $t \in \widetilde{\mathcal{A}}_{(H)}$. Then there exists a sequence $\left(\theta_{n}, t_{n}\right) \in \widetilde{\mathcal{E}}_{(H)}$ whose limit is $(0, t)$. As $\mathcal{E}_{(H)}=(\Pi \times \mathrm{id})^{-1}\left(\widetilde{\mathcal{E}}_{(H)}\right)$, there is a sequence $\left(v_{n}, t_{n}\right) \in \mathcal{E}_{(H)}$ convergent to a point in $\left(\Pi^{-1}(0), t\right)$. Because the action of $G_{q_{e}}$ on $T_{q_{e}} Q$ is linear we have that $\Pi^{-1}(0)=0$ and hence $t \in \mathcal{A}_{(H)}$.

Remark 4.3. If $H$ is conjugated to a proper subgroup of $G_{q_{e}}$ we have $\operatorname{codim}_{\mathbb{R}^{k}} \mathcal{A}_{(H)} \geq 1$.

The next theorem, due to Koenig and Chossat [13, gives a sufficient criterion for the existence of symmetry breaking branches of relative equilibria for the family of vector fields $\overline{X_{\lambda}}$ in a neighborhood of $0_{q_{e}}$.

Theorem 4.4. Let $H$ be an isotropy group conjugate to a proper subgroup of $G_{q_{e}}$ and $\overline{X_{\lambda}} \in \mathcal{C}^{\infty}\left(T_{q_{e}} Q \times\right.$ $\left.\mathbb{R}, T_{q_{e}} Q\right)_{o}^{G_{q_{e}}}$ verifying the transversality condition given in Definition 4.1 . For $v_{q_{e}} \in\left(T_{q_{e}} Q\right)_{(H)}$ define $n_{H}:=\operatorname{rank} N\left(G_{v_{q_{e}}}\right) / G_{v_{q_{e}}}$.

(a) If $\operatorname{codim}_{\mathbb{R}^{k}} \mathcal{A}_{(H)} \geq 2$ then the system $\dot{v}_{q_{e}}=\overline{X_{\lambda}}\left(v_{q_{e}}\right)$ does not have relative equilibria with isotropy conjugate to $H$ in a neighborhood of the origin $0_{q_{e}}$.

(b) If $\operatorname{codim}_{\mathbb{R}^{k}} \mathcal{A}_{(H)}=1$ and $\gamma(0) \in \mathcal{A}_{(H)}$ then there exists a branch of tori of dimension $n_{H}$ bifurcating from $0_{q_{e}}$. The torus corresponding to $\lambda$ is invariant under the flow of $\overline{X_{\lambda}}$. The points on all of these tori have isotropy type $H$.

For the proof of this theorem we will need the following result of Field and Richardson 9 :

Theorem 4.5. Let $H$ be an isotropy group conjugate to a proper subgroup of $G_{q_{e}}$ and $\widetilde{\bar{X}_{\lambda}}$ the projection on the orbit space $\left(T_{q_{e}} Q\right) / G_{q_{e}}$ of the family of vector fields $\overline{X_{\lambda}} \in \mathcal{C}^{\infty}\left(T_{q_{e}} Q \times \mathbb{R}, T_{q_{e}} Q\right)_{o}^{G_{q_{e}}}$ verifying the transversality condition given in Definition 4.1 .

(a) If $\operatorname{codim}_{\mathbb{R}^{k}} \widetilde{\mathcal{A}}_{(H)} \geq 2$ then ${\widetilde{\overline{X_{\lambda}}}}^{-1}([0])$ does not contain points with isotropy conjugate to $H$.

(b) If $\operatorname{codim}_{\mathbb{R}^{k}} \widetilde{\mathcal{A}}_{(H)}=1$ and $\gamma(0) \in \widetilde{\mathcal{A}}_{(H)}$ then there exist a $\mathcal{C}^{1}$ curve $c:[0,1] \mapsto{\widetilde{\bar{X}_{\lambda}}}^{-1}$ ([0]) such that $c(0)=0, c^{\prime}(0) \neq 0$, and all the points on the curve have isotropy conjugate to $H$. 
Remark 4.6. In the above theorem the condition $\overline{X_{\lambda}} \in \mathcal{C}^{\infty}\left(T_{q_{e}} Q \times \mathbb{R}, T_{q_{e}} Q\right)_{o}^{G_{q_{e}}}$ is used to insure that $f_{1}(0,0)=0$ and $\left.\frac{\partial}{\partial \lambda} f_{1}(0, \lambda)\right|_{\lambda=0} \neq 0$, where $f_{1}$ is the first coefficient in the expansions (4.1) or (4.2). In both cases considered here, this is equivalent to $\sigma_{X}(0)=0$ and $\sigma_{X}^{\prime}(0) \neq 0$.

Now we give the proof of Theorem 4.4

Proof. (a) Suppose that the system $\dot{v}_{q_{e}}=\overline{X_{\lambda}}\left(v_{q_{e}}\right)$ admits relative equilibria with isotropy subgroup conjugated to $H$ in a neighborhood of $0_{q_{e}}$. This means that the projection of this vector field has zeroes whose isotropy subgroups are conjugate to $H$. By Theorem 4.5 this implies that $\operatorname{codim}_{\mathbb{R}^{k}} \widetilde{\mathcal{A}}_{(H)} \leq 1$ and $\gamma(0) \in \widetilde{\mathcal{A}}_{(H)}$. This is in contradiction with $\operatorname{codim}_{\mathbb{R}^{k}} \widetilde{\mathcal{A}}_{(H)}=\operatorname{codim}_{\mathbb{R}^{k}} \mathcal{A}_{(H)} \geq 2$. (b) Suppose that $\operatorname{codim}_{\mathbb{R}^{k}} \mathcal{A}_{(H)}=1$ and $\gamma(0) \in \mathcal{A}_{(H)}$. By Proposition 4.2 this implies that $\operatorname{codim}_{\mathbb{R}^{k}} \widetilde{\mathcal{A}}_{(H)}=1$ and $\gamma(0) \in \widetilde{\mathcal{A}}_{(H)}$. Theorem 4.5 guarantees the existence of a $\mathcal{C}^{1}$ curve $c: \lambda \in[0,1] \mapsto{\widetilde{\bar{X}_{\lambda}}}^{-1}([0]), c(0)=0$, $c^{\prime}(0) \neq 0$, all of whose points have isotropy subgroup conjugate to $H$. The inverse image of this curve under the orbit space projection $\Pi$ is the $G_{q_{e}}$-orbit of a branch of relative equilibria $v_{\lambda}$. For a fixed $\lambda$, using Krupa's Theorem [14, we decompose locally in a tubular neighborhood of the orbit $G_{q_{e}} \cdot v_{\lambda}$, the $G_{q_{e}}$-equivariant vector field $\overline{X_{\lambda}}$ into its tangential and normal components. Since the projection of this vector field to the orbit space has $c(\lambda)$ as equilibrium we have that $\overline{X_{\lambda}}\left(v_{\lambda}\right) \in \mathfrak{g}_{q_{e}} \cdot v_{\lambda}$. This means that the local normal component of the vector field vanishes. So, the solution of the Cauchy problem $\dot{w}_{q_{e}}=\overline{X_{\lambda}}\left(w_{q_{e}}\right), w_{q_{e}}(0)=v_{\lambda}$, is given by $w_{q_{e}}(t)=g(t) \cdot v_{\lambda}$, where $g(t)=\exp \left(\xi_{\lambda} t\right)$, for small enough $t \geq 0$ and $\xi_{\lambda} \in \mathfrak{g}_{q_{e}}$. The existence for each $\lambda$ of a flow invariant maximal torus of rank $n_{H}$ on which the solution $\exp \left(\xi_{\lambda} t\right) \cdot v_{\lambda}$ is generically dense is guaranteed by Theorem 4.1 in 14. Hence we have obtained a branch $w_{q_{e}}^{\lambda}(t)=\exp \left(\xi_{\lambda} t\right) \cdot v_{\lambda}$ of periodic or quasi-periodic solutions all of whose points have isotropy type conjugate to $H$.

Using Proposition 3.6 we can deduce the same result as the one in Theorem 4.4 for a one-parameter family of vector fields $X_{\lambda}$ defined in a small neighborhood around the point $q_{e} \in Q$. The resulting bifurcation result will give a family of tori bifurcating from $q_{e}$ whose symmetry is broken to that of the a priori given subgroup $H \subsetneq G_{q_{e}}$. The bifurcating solutions are periodic or quasi-periodic curves lying on these tori. The method given by Proposition 3.6 allows one to always translate bifurcation results obtained in the linear setting to analogous theorems on the manifolds itself.

In the case of the Hopf bifurcation, several additional remarks are in order. First, the symmetry group of the problem can be considered to be of the form $G \times S^{1}$. This follows from a normal form result (see e.g. [10, Chapter XVI) which ensures that there is a natural $S^{1}$-action on $T_{q_{e}} Q$ such that the family of vector fields $\overline{X_{\lambda}}$ are $G \times S^{1}$-equivariant. Second, given $k \in \mathbb{N}$ and a $G$-equivariant family of vector fields $\overline{X_{\lambda}}$ on $T_{q_{e}} Q$, there is a $\mathcal{C}^{k} \lambda$-dependent change of variables on $T_{q_{e}} Q$ that maps $\overline{X_{\lambda}}$ into new family of vector fields $\widetilde{X_{\lambda}}$ which is $G \times S^{1}$-equivariant and that coincides with $\overline{X_{\lambda}}$ up to order $k$. Third, one can interpret this result as saying that close enough to $0_{q_{e}}$ the $S^{1}$-relative equilibria of the $G \times S^{1}$ - equivariant family $\widetilde{X_{\lambda}}$ are mapped by this change of variables to the set of periodic solutions of $\overline{X_{\lambda}}$. Similarly, the $G \times S^{1}$-relative equilibria of $\widetilde{X_{\lambda}}$ are mapped by this change of variables to $G$-relative periodic orbits of $\overline{X_{\lambda}}$.

\section{Hamiltonian Steady State and Hamiltonian Hopf Bifurcation with Symmetry}

In this section we study a bifurcation problem similar to the one in Theorem 4.4 in the context of a Hamiltonian system on a symplectic vector space. To obtain the symmetry breaking result the Hamiltonian nature of the vector field plays a crucial role. One cannot deduce Theorem 5.3 below from the generic results in [13] because the non-degeneracy conditions are not satisfied. 
Let $(V, \omega)$ be a symplectic vector space on which the compact Lie group $G$ acts linearly and symplectically. In this case it is known that the map $G \times V \rightarrow V$ is smooth. Let $F_{\lambda}: V \rightarrow \mathbb{R}, \lambda \in \mathbb{R}$, be a family of smooth $G$-invariant functions. If $0 \in V$ is an equilibrium (with symmetry $G$ ) of the Hamiltonian vector field $X_{F_{0}}$, we are interested in finding conditions that guarantee the existence of smooth branches of tori bifurcating from zero such that all the points on these tori have symmetry conjugate to an a priori fixed proper symmetry subgroup $H$. Analogous to the cases considered in the previous section we shall analyze here the following situations:

- Hamiltonian steady state bifurcation with symmetry:

$$
D X_{F_{\lambda}}(0)=\sigma(\lambda) \mathbb{J}
$$

where $\sigma:(-\epsilon, \epsilon) \rightarrow \mathbb{R}$ is a smooth map satisfying $\sigma(0)=0$ and $\sigma^{\prime}(0) \neq 0$. In addition, it is assumed that the vector space $V$ carries a natural complex structure $\mathbb{J}$ and that the compact Lie group $G$ acts on this complex vector space $V$ irreducibly (as a complex representation). In this case a basis for $\mathfrak{g l}(V)^{G}$, the endomorphisms of $V$ which commute with the $G$-action, is formed by $\{\mathrm{I}, \mathbb{J}\}$. This implies that a symplectic matrix in $\mathfrak{g l}(V)^{G}$ should be a real multiple of $\mathbb{J}$. In this case, we have passing of eigenvalues of $D X_{F_{\lambda}}(0)$ on the imaginary axis at the origin as $\lambda$ crosses zero.

- Hamiltonian Hopf bifurcation with symmetry:

$$
D X_{F_{\lambda}}(0)=\sigma(\lambda) \mathrm{A}_{1}+\rho(\lambda) \mathrm{A}_{2}+\tau(\lambda) \mathrm{A}_{3}+\psi(\lambda) \mathrm{A}_{4}
$$

where

$$
\begin{array}{ll}
\mathrm{A}_{1}=\left[\begin{array}{cc}
\mathbf{0} & \mathbf{0} \\
\mathrm{I} & \mathbf{0}
\end{array}\right], & \mathrm{A}_{2}=\left[\begin{array}{ll}
\mathbf{0} & \mathrm{I} \\
\mathbf{0} & \mathbf{0}
\end{array}\right], \\
\mathrm{A}_{3}=\left[\begin{array}{cc}
\mathrm{I} & \mathbf{0} \\
\mathbf{0} & -\mathrm{I}
\end{array}\right], & \mathrm{A}_{4}=\left[\begin{array}{ll}
\mathbb{J} & \mathbf{0} \\
\mathbf{0} & \mathbb{J}
\end{array}\right] .
\end{array}
$$

and $\sigma, \rho, \tau, \psi:(-\epsilon, \epsilon) \rightarrow \mathbb{R}$ are smooth maps satisfying $\sigma(0)=0, \sigma^{\prime}(0) \neq 0, \rho(0)=-1, \tau(0)=0$ and $\psi(0)=-\nu_{0}$, with $\nu_{0}>0$. In addition, it is assumed that the vector space $V=V_{0} \oplus V_{1}$, where $V_{0}$ and $V_{1}$ are isomorphic complex dual irreducible representations of the compact Lie group $G$. The compact Lie group $G$ is considered to be of the form $G=\Gamma \times S^{1}$. See for example 6 .

In the following will denote by $\mathcal{C}^{\infty}(V \times \mathbb{R}, V)_{\text {oo }}^{G}$ the class of $G$-equivariant Hamiltonian vector fields that verifies one of the above non-degeneracy conditions.

As opposed to the general case, if the vector field is Hamiltonian, it turns out that one needs to use only invariant polynomials, as the proposition below shows.

Proposition 5.1. Let $(V, \omega)$ be a symplectic representation space of the compact Lie group $G$. Denote by $\left\{\theta_{1}, \theta_{2}, \ldots, \theta_{l}\right\}$ a set of generators of $\mathcal{P}_{G}(V)$. If $F: V \rightarrow \mathbb{R}$ is a smooth $G$-invariant function then the associated Hamiltonian vector field $X_{F}$ has the following expression in canonical coordinates, in terms of invariant polynomials and their gradients:

$$
X_{F}(x)=\sum_{i=1}^{l} \frac{\partial \tilde{F}}{\partial \theta_{i}}\left(\theta_{1}(x), \ldots, \theta_{l}(x)\right) \mathbb{J} \operatorname{grad}_{x}\left(\theta_{i}\right)(x), \quad \text { for any } \quad x \in V,
$$

where $\tilde{F}: \mathbb{R}^{l} \rightarrow \mathbb{R}$ is given by:

$$
F(x)=\tilde{F}\left(\theta_{1}(x), \ldots, \theta_{l}(x)\right), \quad \text { for any } \quad x \in V .
$$


Proof. The proof follows directly from the definition of the Hamiltonian vector field.

The next step gives a method for projecting a $G$-equivariant Hamiltonian vector field on the orbit space. To do this, let $F$ be a $G$-invariant smooth function and $X_{F}$ its associated Hamiltonian vector field. Therefore, $X_{F}$ is $G$-equivariant on $(V, \omega)$. Note that $\mathbb{J} \operatorname{grad} \theta_{i}$ are equivariant polynomials. Thus, by the proposition above we can write $X_{F}$ in terms of invariants and equivariants as

$$
X_{F}(x)=\sum_{i=1}^{l} \frac{\partial \tilde{F}}{\partial \theta_{i}}\left(\theta_{1}(x), \ldots, \theta_{l}(x)\right) \mathbb{J} \operatorname{grad}_{x} \theta_{i}(x), \quad \text { for any } \quad x \in V,
$$

where the function $\tilde{F}$ is smooth on $\mathbb{R}^{l}$. To obtain the projection of this vector field on the orbit space $V / G$, one has to compute the derivatives of the invariant generators $\theta_{j}$ with respect to time:

$$
\begin{aligned}
\dot{\theta}_{j} & =\mathbf{d} \theta_{j}(x)(\dot{x})=\left(\operatorname{grad}_{x}\left(\theta_{j}\right)\right)^{t} \cdot\left(\sum_{i=1}^{l} \frac{\partial \tilde{F}}{\partial \theta_{i}} \mathbb{J} \operatorname{grad}_{x}\left(\theta_{i}\right)\right) \\
& =\sum_{i=1}^{l} \frac{\partial \tilde{F}}{\partial \theta_{i}}\left\{\theta_{j}, \theta_{i}\right\} .
\end{aligned}
$$

The projection $\widetilde{X}_{F}$ of $X_{F}$ on the orbit space $V / G$ has thus the expression

$$
\widetilde{X}_{F}(\theta)=\mathcal{P}(\theta) \operatorname{grad}_{\theta}(\tilde{F})
$$

where

$$
\mathcal{P}(\theta):=\left[\begin{array}{cccc}
0 & \left\{\theta_{1}, \theta_{2}\right\} & \ldots & \left\{\theta_{1}, \theta_{l}\right\} \\
\ldots & \cdots & \cdots & \cdots \\
\left\{\theta_{l}, \theta_{1}\right\} & \cdots & \cdots & 0
\end{array}\right] .
$$

As in the Riemannian case, one search for symmetry breaking zeroes of the projected vector field. To do this, we need a few preliminary results. Define the following maps associated to the family of smooth $G$-invariant functions $F: V \times \mathbb{R} \rightarrow \mathbb{R}$

$$
\begin{gathered}
\widetilde{\Phi}: V / G \times \mathbb{R}^{l} \ni(\theta, t) \mapsto \sum_{i=1}^{l} t_{i}\left[\begin{array}{c}
\left\{\theta_{1}, \theta_{i}\right\} \\
\left\{\theta_{2}, \theta_{i}\right\} \\
\cdots \\
\left\{\theta_{l}, \theta_{i}\right\}
\end{array}\right] \in V / G \subset \mathbb{R}^{l} \\
\gamma: \mathbb{R} \ni \lambda \mapsto\left(\frac{\partial \tilde{F}}{\partial \theta_{1}}(0, \lambda), \ldots, \frac{\partial \tilde{F}}{\partial \theta_{l}}(0, \lambda)\right) \in \mathbb{R}^{l}
\end{gathered}
$$

and the sets

$$
\begin{gathered}
\widetilde{\mathcal{E}}=\left\{(\theta, t) \in V / G \times \mathbb{R}^{l} \mid \widetilde{\Phi}(\theta, t)=0\right\} \\
\widetilde{\mathcal{E}}_{(H)}=\left\{(\theta, t) \in \widetilde{\mathcal{E}} \mid \theta \in \Pi\left(V_{(H)}\right)\right\} \\
\widetilde{\mathcal{A}}_{(H)}=\overline{\widetilde{\mathcal{E}}_{(H)}} \cap\left(\{[0]\} \times \mathbb{R}^{l}\right),
\end{gathered}
$$

where $\Pi: V \rightarrow V / G$ is the orbit space projection and $H \subset G$ is an isotropy subgroup of the $G$-action on $V$. Recall that $\widetilde{\mathcal{E}}$ is a semialgebraic subset of $\mathbb{R}^{2 l}$. Denote by $\Sigma$ the canonical Whitney stratification of $\widetilde{\mathcal{E}}$. This stratification induces a Whitney semialgebraic stratification $\Sigma_{(H)}$ on each $\widetilde{\mathcal{E}}_{(H)}$. In particular, $\Sigma_{G}$ stratifies $\widetilde{\mathcal{E}}_{(G)}=\{[0]\} \times \mathbb{R}^{l}$. Next we recall the notion of $G$-transversality introduced by Konig and Chossat [13] that was in turn inspired by Bierstone [4] and Field [8]; see Definition 4.1] In the present case, this says that the projection to the orbit space $V / G$ of a $G$-equivariant smooth family of vector 
fields $\tilde{X}_{F_{\lambda}} \in \mathfrak{X}(V)$ is transverse to $[0] \in V / G$ at $\lambda=0 \in \mathbb{R}$ if $\gamma: \mathbb{R} \rightarrow \mathbb{R}^{l}$ is transverse at $\lambda=0$ to the stratum of $\Sigma_{G}$ containing $\gamma(0)$, that is, im $T_{0} \gamma+T_{\gamma(0)} S=\mathbb{R}^{l}$, where $S$ is the stratum of $\Sigma_{G}$ containing $\gamma(0)$. Analogous to the map (5.3) we define

$$
\Phi: V \times \mathbb{R}^{l} \ni(x, t) \mapsto \sum_{i=1}^{l} t_{i} \mathbb{J} \operatorname{grad}_{x} \theta_{i}(x) \in V .
$$

This map induces the following sets parameterized by the isotropy type subgroups of the $G$-action on $V$ :

$$
\begin{gathered}
\mathcal{E}_{(H)}=\left\{(x, t) \in V_{(H)} \times \mathbb{R}^{l} \mid \Phi(x, t) \in \mathfrak{g} \cdot x\right\}, \\
\mathcal{A}_{(H)}=\overline{\mathcal{E}_{(H)}} \cap\left(\{0\} \times \mathbb{R}^{l}\right) .
\end{gathered}
$$

The sets $\mathcal{E}_{(H)}$ and $\mathcal{A}_{(H)}$ are semialgebraic sets in $V \times \mathbb{R}^{l}$ and $\mathbb{R}^{l}$, respectively. A proof analogous to that of Proposition 4.2 shows that $\mathcal{E}_{(H)}=(\Pi \times \mathrm{id})^{-1}\left(\widetilde{\mathcal{E}}_{(H)}\right)$ and $\widetilde{\mathcal{A}}_{(H)}=\mathcal{A}_{(H)}$.

We shall prove below that if $H \neq G$, then $\operatorname{codim}_{\mathbb{R}^{l}} \mathcal{A}_{(H)} \geq 1$.

Theorem 5.2. Let $V$ be a symplectic representation space of a compact Lie group $G$ and $\left\{\theta_{1}, \ldots, \theta_{l}\right\}$ a Hilbert base. Suppose there exist $i_{0}, i_{1} \in\{1, \ldots, l\}, i_{0} \neq i_{1}, D_{i_{0}, i_{1}}:=\left\{x \in V \mid\left\{\theta_{i_{0}}, \theta_{i_{1}}\right\}(x) \neq 0\right\} \neq \varnothing$, and $x_{0} \in V$ such that $\lim _{x \rightarrow x_{0}} \frac{\left\{\theta_{i_{0}}, \theta_{i}\right\}(x)}{\left\{\theta_{i_{0}}, \theta_{i_{1}}\right\}(x)}=0$, for any $i \in\{1, \ldots, l\} \backslash\left\{i_{1}\right\}$. Let $H$ be an isotropy subgroup of the $G$-representation on $V$. If $(H) \neq(G)$ then we have $\widetilde{\mathcal{A}}_{(H)} \subset\left(\mathbb{R}^{1}\right)^{\perp}=\left\{t \in \mathbb{R}^{l} \mid t_{i_{1}}=0\right\}$ and $\widetilde{\mathcal{A}}_{(G)}=\mathbb{R}^{l}$. In particular, $\operatorname{codim}_{\mathbb{R}^{l}} \widetilde{\mathcal{A}}_{(H)} \geq 1$.

Proof. From (5.3), it follows that the $i_{0}$-component of the map $\widetilde{\Phi}$ is given by

$$
t_{1}\left\{\theta_{i_{0}}, \theta_{1}\right\}+t_{2}\left\{\theta_{i_{0}}, \theta_{2}\right\}+\cdots+t_{l}\left\{\theta_{i_{0}}, \theta_{l}\right\} .
$$

Let $\theta:=\left(\theta_{1}, \ldots, \theta_{l}\right) \in V / G \subset \mathbb{R}^{l}$. If $(\theta, t) \in \widetilde{\mathcal{E}}_{(H)}$, the above relation becomes

$$
t_{1}\left\{\theta_{i_{0}}, \theta_{1}\right\}+t_{2}\left\{\theta_{i_{0}}, \theta_{2}\right\}+\cdots+t_{l}\left\{\theta_{i_{0}}, \theta_{l}\right\}=0 .
$$

This implies that

$$
t_{1}\left\{\theta_{i_{0}}, \theta_{1}\right\}(x)+t_{2}\left\{\theta_{i_{0}}, \theta_{2}\right\}(x)+\cdots+t_{l}\left\{\theta_{i_{0}}, \theta_{l}\right\}(x)=0, \quad(\forall) x \in D_{i_{0}, i_{1}} .
$$

Dividing this relation by $\left\{\theta_{i_{0}}, \theta_{i_{1}}\right\}(x)$ and letting $x \rightarrow x_{0}$ one concludes that $t_{i_{1}}=0$. This proves that $\widetilde{\mathcal{A}}_{(H)} \subset\left\{t \in \mathbb{R}^{l} \mid t_{i_{1}}=0\right\}$. The identity $\widetilde{\mathcal{A}}_{(G)}=\mathbb{R}^{l}$ follows directly from the definitions.

Adapting the Proposition 5.16 of Field and Richardson 9] to the family of vector fields $X_{F_{\lambda}}$, yields the following result. Note that $X_{F_{\lambda}} \in \mathcal{C}^{\infty}(V \times \mathbb{R}, V)_{\text {oo }}^{G}$ implies the condition from the Remark 4.6] this condition being equivalent to $\sigma(0)=0$ and $\sigma^{\prime}(0) \neq 0$.

Theorem 5.3. Let $V$ be a symplectic representation space of a compact Lie group $G$ and $\left\{\theta_{1}, \ldots, \theta_{l}\right\}$ a Hilbert base. Suppose there exist $i_{0}, i_{1} \in\{1, \ldots, l\}, i_{0} \neq i_{1}, D_{i_{0}, i_{1}}:=\left\{x \in V \mid\left\{\theta_{i_{0}}, \theta_{i_{1}}\right\}(x) \neq 0\right\} \neq \varnothing$, and $x_{0} \in V$ such that $\lim _{x \rightarrow x_{0}} \frac{\left\{\theta_{i_{0}}, \theta_{i}\right\}(x)}{\left\{\theta_{i_{0}}, \theta_{i_{1}}\right\}(x)}=0$, for any $i \in\{1, \ldots, l\} \backslash\left\{i_{1}\right\}$. Let $H$ be a given proper isotropy subgroup of $G$ and assume that the family of Hamiltonian vector fields $X_{F_{\lambda}} \in \mathcal{C}^{\infty}(V \times \mathbb{R}, V)_{\text {oo }}^{G}$ satisfies the transversality condition given in Definition 4.1. For $x \in V_{(H)}$ define $n_{H}:=\operatorname{rank} N\left(G_{x}\right) / G_{x}$.

(a) If $\operatorname{codim}_{\mathbb{R}^{l}} \mathcal{A}_{(H)} \geq 2$ then the system $\dot{x}=X_{F_{\lambda}}(x)$ does not have relative equilibria with isotropy conjugate to $H$ in a neighborhood of the origin 0 .

(b) If $\operatorname{codim}_{\mathbb{R}^{l}} \mathcal{A}_{(H)}=1$ and $\gamma(0) \in \mathcal{A}_{(H)}$ then there exists a branch of tori of dimension $n_{H}$, formed by points of isotropy type $H$, bifurcating from 0 . The torus corresponding to $\lambda$ is invariant under the flow of $X_{F_{\lambda}}$. 
We shall make some remarks regarding the Hamiltonian Hopf bifurcation. We have made the hypothesis that the symmetry group of the problem is of the form $G \times S^{1}$. This always holds if we assume only that the system has a compact Lie group $G$ of symmetries represented on the symplectic vector space $V$. The reason for this is that in the case of the Hamiltonian Hopf bifurcation there is a natural $S^{1}$ action on $V$ given by the exponential of the semisimple part of the linearization on the resonance space corresponding to $\nu_{0}$. More precisely, let $\left(V, \omega, F_{\lambda}\right)$ be a $\lambda$-parameter family $(\lambda \in \mathbb{R}$ in a neighborhood of the origin) of $G$-Hamiltonian systems such that $F_{0}(0)=0, \mathbf{d} F_{0}(0)=0$, and the $G$-equivariant infinitesimally symplectic linear map $D X_{F_{0}}(0)$ is nonsingular and has $\pm i \nu_{0}$ as eigenvalues. Let $\left(U_{\nu_{0}},\left.\omega\right|_{U_{\nu_{0}}}\right)$ be the resonance space of $D X_{F_{0}}(0)$ with primitive period $T_{\nu_{0}}:=2 \pi / \nu_{0}$. Recall that the resonance space is the direct sum of the real eigenspaces of $D X_{F_{0}}(0)$ corresponding to eigenvalues of the form $\pm k i \nu_{0}$ with $k=1,2, \ldots$ A theorem of Vanderbauwhede and van der Meer [26] states that for each $k \geq 0$ one can find a $C^{k}$-mapping $\psi: U_{\nu_{0}} \times \mathbb{R} \rightarrow V$ and a $C^{k+1}$-function $\widehat{F_{\lambda}}: U_{\nu_{0}} \times \mathbb{R} \rightarrow \mathbb{R}$ such that $\psi(0, \lambda)=0$, for all $\lambda, D_{U_{\nu_{0}}} \psi(0,0)=\mathbb{I}_{U_{\nu_{0}}}$, and $\widehat{F_{\lambda}}$ is a $G \times S^{1}$-invariant function that coincides with $F_{\lambda}$ up to order $k+1$. In the same paper it is shown (Theorem 3.2) that if $\lambda$ is close to 0 then the $S^{1}$-relative equilibria

of the $G \times S^{1}$-invariant Hamiltonian $\widehat{F_{\lambda}}$ that lie in a sufficiently small neighborhood of the origin in $U_{\nu_{0}}$ are mapped by $\psi(\cdot, \lambda)$ to the set of periodic solutions of $\left(V, \omega, F_{\lambda}\right)$ in a neighborhood of $0 \in V$ with periods close to $T_{\nu_{0}}$. Thus, on can replace the search of periodic orbits of $\left(V, \omega, F_{\lambda}\right)$ by the search of $S^{1}$-relative equilibria of the $G \times S^{1}$-invariant family of Hamiltonian systems $\left(U_{\nu_{0}},\left.\omega\right|_{U_{\nu_{0}}}, \widehat{F_{\lambda}}\right)$. Similarly, the $G \times S^{1}$-relative equilibria of $X_{\widehat{F_{\lambda}}}$ are mapped by $\psi(\cdot, \lambda)$ to $G$-relative periodic orbits of $X_{F_{\lambda}}$.

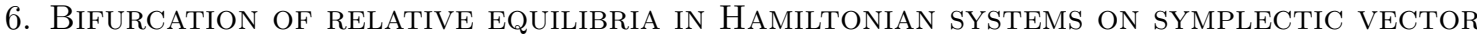 SPACES}

In this section we will prove a bifurcation result in symplectic vector spaces in the context of Hamiltonian systems. We consider a symplectic representation space $(V, \omega)$ of a compact Lie group $G$ and let $\left\{\theta_{1}, \ldots, \theta_{l}\right\}$ a Hilbert base. We will denote by $V_{(H)} \subset V$ the principal stratum of the action of $G$ on $V$ and by $\Pi: V \rightarrow V / G$ the orbit space projection. Recall that each connected component of $V_{(H)}$ is an open dense subset of the vector space $V$. We consider a family of $G$-invariant smooth functions $F_{\lambda}: V \rightarrow \mathbb{R}, \lambda \in \mathbb{R}$, such that there exists $\lambda_{0} \in \mathbb{R}$ and $v_{0} \in V_{(H)}$ a relative equilibrium for the Hamiltonian vector field $X_{F_{\lambda_{0}}}$. We will prove that under a certain non-degeneracy condition we have the existence of a smooth branch of relative equilibria (starting from $v_{0}$ ) for the Hamiltonian vector fields $X_{F_{\lambda}}$ for $\lambda$ close to $\lambda_{0}$. To do this we recall some of the techniques necessary to find the expression of the projected Hamiltonian vector field on $V / G$. More precisely, if $F_{\lambda}(x)=\tilde{F}_{\lambda}\left(\theta_{1}(x), \ldots, \theta_{l}(x)\right)$, for any $x \in V$, where $\tilde{F}_{\lambda}: \mathbb{R}^{l} \rightarrow \mathbb{R}$ is smooth, then the $j$-component of the projection on the orbit space $V / G$ of the Hamiltonian vector field $X_{F_{\lambda}}$ is given by:

$$
\sum_{i=1}^{l} \frac{\partial \tilde{F}_{\lambda}}{\partial \theta_{i}}\left\{\theta_{j}, \theta_{i}\right\} .
$$

Searching for relative equilibria of a vector field is the same as searching for equilibria for the projected vector field on the orbit space. Thus, we reduced the problem to find branches in $\lambda$ of solutions of the system:

$$
\sum_{i=1}^{l} \frac{\partial \tilde{F}_{\lambda}}{\partial \theta_{i}}\left(\theta_{1}, \ldots, \theta_{l}\right)\left\{\theta_{j}, \theta_{i}\right\}=0, \quad \text { for } \quad j \in\{1, \ldots, l\}
$$


To do this, we introduce the function $g:\left(V / G \subset \mathbb{R}^{l}\right) \times \mathbb{R} \rightarrow \mathbb{R}^{l}, g=\left(g_{1}, \ldots, g_{l}\right)$, where the components $g_{j}$ are defined by:

$$
g_{j}\left(\left(\theta_{1}, \ldots, \theta_{l}\right), \lambda\right):=\sum_{i=1}^{l} \frac{\partial \tilde{F}_{\lambda}}{\partial \theta_{i}}\left(\theta_{1}, \ldots, \theta_{l}\right)\left\{\theta_{j}, \theta_{i}\right\}, \quad \text { for } \quad j \in\{1, \ldots, l\} .
$$

As $v_{0} \in V_{(H)}$ is a relative equilibrium of $X_{F_{\lambda_{0}}}$ this implies that $\theta_{0}=: \Pi\left(v_{0}\right)$ is an equilibrium point for the projection on the orbit space $V / G \subset \mathbb{R}^{l}$ of the Hamiltonian vector field $X_{F_{\lambda_{0}}}$. This means that $\theta_{0} \in V / G \subset \mathbb{R}^{l}$ is a solution of the equation $g\left(\theta, \lambda_{0}\right)=0$. The next theorem will give a sufficient condition that guarantees the existence of a smooth curve of solutions of the equation $g(\theta, \lambda)=0$, in an open neighborhood of the point $\left(\theta_{0}, \lambda_{0}\right)$.

Theorem 6.1. Let $(V, \omega)$ be a symplectic representation space of a compact Lie group $G$ and $\Pi: V \rightarrow$ $V / G$ be the orbit space projection. Let $F_{\lambda}: V \rightarrow \mathbb{R}, \lambda \in \mathbb{R}$, be a family of $G$-invariant smooth functions. Suppose that $v_{0} \in V$ is a relative equilibrium of the Hamiltonian vector field $X_{F_{\lambda_{0}}}$ which belongs to the principal stratum of the $G$-action on $V$. Denote $\theta_{0}=: \Pi\left(v_{0}\right)$. If $\mathbf{d}_{\theta} g\left(\theta_{0}, \lambda_{0}\right)$ is non-degenerate, then there exists an open subset $I$ of $\mathbb{R}$ containing $\lambda_{0}$ and a smooth curve $\gamma: \lambda \in I \mapsto \gamma(\lambda) \in V / G \subset \mathbb{R}^{l}$, such that $\gamma(\lambda) \in V / G \subset \mathbb{R}^{l}$ lifts to relative equilibria of $X_{F_{\lambda}}$ and $\gamma\left(\lambda_{0}\right)=\theta_{0} \in V / G \subset \mathbb{R}^{l}$.

Proof. The proof is a consequence of the implicit function theorem and of the fact that $v_{0} \in V_{(H)} \subset V$ is an element of the principal stratum; the connected component containing it is an open subset of $V$.

Note that in this case the only thing we can say about the symmetry of the relative equilibria on the branch is that for $\lambda$ small enough all of them are of principal type. This theorem can be used in the following way to study bifurcating branches of relative equilibria from a given one with isotropy $K$. Let $v_{0} \in V$ be a relative equilibrium with isotropy $K$. Since $G$ is compact, so is $K$ and hence the subspace $V^{K}$ of $K$-fixed vectors is a symplectic subspace of $(V, \omega)$. Let us assume that the normalizer $N(K)$ of $K$ in $G$ is closed, which implies that $N(K) / K$ is a compact Lie group that acts naturally on $V^{K}$. Note that the restriction of this action on the $K$-invariant open subset $V_{K}$ is free. Then we can apply the previous theorem to $V^{K}, N(K) / K$, and the restrictions of the vector fields $X_{F_{\lambda}}$ to the open subset $V_{K}$ of $V^{K}$. The corresponding function $g$ from before will be restricted to the open set $V_{K} /(N(K) / K)$ and, under the same non-degeneracy hypothesis, we obtain $\lambda$-branches of relative equilibria for $X_{F_{\lambda}}$ consisting of points with isotropy exactly $K$.

\section{From Symplectic VeCtor SPACES to Manifolds}

In this short section we will give a method of translating the results obtained in the previous two sections from symplectic vector spaces to symplectic manifolds. As all the symmetry breaking results in sections 5 and 6 are of local type we will make use of the Equvariant Darboux Theorem to pass locally from a symplectic manifold to a symplectic vector space.

Theorem 7.1. Suppose that $\Gamma$ is a compact Lie group acting on a finite-dimensional symplectic manifold $Q$ and let $\omega$ be a $\Gamma$-invariant symplectic form on $Q$. Let $q \in Q$ be a $\Gamma$-invariant point. Then there are open $\Gamma$-invariant neighborhoods $U \subset Q$ of $q$ and $V \subset T_{q} Q$ of $0_{q} \in T_{q} Q$ and a $\Gamma$-invariant symplectic diffeomorphism $f:(U, \omega) \rightarrow(V, \omega(q))$, such that $f(q)=0_{q}$.

This theorem follows from the $G$-relative Darboux Theorem (see, for example, [20]).

Theorem 7.2. Let $Q$ be a smooth manifold and $\omega_{0}$ and $\omega_{1}$ two symplectic forms on it. Let $G$ be a Lie group acting properly on $Q$ and symplectically with respect to both $\omega_{0}$ and $\omega_{1}$. Assume that

$$
\omega_{0}(g \cdot q)\left(v_{g \cdot q}, w_{g \cdot q}\right)=\omega_{1}(g \cdot q)\left(v_{g \cdot q}, w_{g \cdot q}\right)
$$


for all $g \in G$ and $v_{g \cdot q}, w_{g \cdot q} \in T_{g \cdot q} Q$. Then there exist two open $G$-invariant neighborhoods $U_{0}$ and $U_{1}$ of $G \cdot q$ and a G-equivariant diffeomorphism $\Psi: U_{0} \rightarrow U_{1}$ such that $\left.\Psi\right|_{G \cdot q}=\operatorname{Id}$ and $\Psi^{*} \omega_{1}=\omega_{0}$.

Theorem 7.1 is proved as follows. On the symplectic manifold $(Q, \omega)$ consider around the point $q$ an open neighborhood $W \subset Q$ contained in a Darboux chart. Thus, on this neighborhood the symplectic form $\omega_{1}$ is the pull back of the symplectic form $\omega(q)$ on $T_{q} Q$. Recall that $\omega(q)$ is $\Gamma$-invariant, where $\Gamma$ acts by linearization of the original action on $T_{q} Q$. Since the point $q$ is $\Gamma$-invariant we can shrink the neighborhood $W$ to a $\Gamma$ invariant open neighborhood $B \subset W$ of $q$. So, on the $\Gamma$-invariant manifold $B$ we have two $\Gamma$-invariant symplectic forms: $\omega$ and $\omega_{1}$. Now using the $\Gamma$-relative Darboux theorem we get the existence of two open $\Gamma$-invariant neighborhoods $U_{0} \subset B$ and $U_{1} \subset B$ of $q$ and a $\Gamma$-equivariant diffeomorphism $\Psi: U_{0} \rightarrow U_{1}$ such that $\left.\Psi\right|_{\Gamma \cdot q}=\operatorname{Id}$ and $\Psi^{*} \omega=\omega_{1}$.

The nonlinear version of the results from sections 5 and 6 can be stated in the following theorem.

Theorem 7.3. Let $G$ be a Lie group acting properly on a symplectic finite dimensional manifold $(Q, \omega)$. Let $F_{\lambda}: Q \rightarrow \mathbb{R}, \lambda \in \mathbb{R}$, be a family of $G$-invariant smooth functions and $q_{e} \in Q$ be a relative equilibrium of the Hamiltonian vector field $X_{F_{\lambda_{0}}}$. Then, in the conditions of Theorems 5.3 and [6.1 the same conclusions hold on the manifold $Q$ around the point $q_{e}$.

Proof. The proof is a consequence of Theorem 7.1 by considering the compact Lie group $G_{q_{e}}$ acting on the symplectic manifold $(Q, \omega)$.

\section{REFERENCES}

[1] R. Abraham and J.E. Marsden, Foundations of Mechanics, Second edition, Addison-Wesley, 1979.

[2] R. Abraham, J.E. Marsden, and T.S. Ratiu, Manifolds, Tensor Analysis, and Applications, Applied Mathematical Sciences, vol. 75, Springer-Verlag, 1988.

[3] M. Abud and G. Sartori, The geometry of spontaneous symmetry breaking, Ann. Phys., 150 (1983), 307-372.

[4] E. Bierstone, General position of equivariant maps, Tans. Amer. Math. Soc., 234(2)(1977), pp. 447-466.

[5] G.E. Bredon, Introduction to Compact Transformation Groups, Academic Press, 1972.

[6] P. Chossat, J.-P. Ortega, and T. S. Ratiu, Hamiltonian Hopf bifurcation with symmetry, Arch. Rat. Mech. Anal., 163 (2002), 1-33, 167 (2003), 83-84.

[7] J.J. Duistermaat and J.A. Kolk, Lie Groups, Universitext, Springer-Verlag, 1999.

[8] M.J. Field, Transversality in G-manfolds, Tans. Amer. Math. Soc., 231(2)(1977), pp. 429-450.

[9] M. J. Field and R. W. Richardson, Symmetry breaking and the maximal isotropy conjecture for reflection groups, Arch. Rat. Mech. Anal., 105(1), 1989, 61-94.

[10] M. Golubitsky, I. Stewart, and D. G. Schaeffer, Singularities and Groups in Bifurcation Theory, Volume II, Volume 69 of Applied Math. Sciences, Springer-Verlag 1988.

[11] K. Kawakubo, The Theory of Transformations Groups, Oxford University Press, 1991.

[12] M. Kensig, Une exploration des espace d'orbites des groupes de Lie compacts et de leurs applications à létude de bifrcations avec symétrie, Ph.D. Thesis, Institut Non Linéaire de Nice, 1995.

[13] M. Kennig and P. Chossat, Caractérisation des bifurcations pour les champs de vecteurs équivariants sous l'action d'un groupe de Lie compact. C. R. Acad. Sci. Paris Sr. I Math., 318(1)(1994): 31-36.

[14] M. KRupa, Bifurcations of relative equilibria, SIAM J. Math. Anal., 21(6), 1990, 1453-1486.

[15] P. Libermann and C.-M. Marle, Symplectic Geometry and Analytical Mechanics, Reidel, 1987.

[16] J.E. Marsden, Lectures on Geometric Methods in Mathematical Physics, Vol 37, SIAM, Philadelphia, 1981.

[17] J.E. Marsden Lectures on Mechanics, London Mathematical Society Lecture Note Series, Volume 174, Cambridge University Press, 1992.

[18] J.E. Marsden and T.S. Ratiu, Introduction to Mechanics and Symmetry, second edition, Volume 17 of Texts in Applied Mathematics, Springer-Verlag, 1999.

[19] J.E. Marsden and A. Weinstein, Reduction of symplectic manifolds with symmetry, Rep. Math. Phys., 5(1974), pp. 121-130.

[20] J.-P. Ortega And T.S. Ratiu, Momentum Maps and Hamiltonian Reduction, Progress in Mathematics, Volume 222, Birkhäuser, 2003.

[21] G. Patrick, Two Axially Symmetric Coupled Rigid Bodies: Relative Equilibria, Stability, Bifurcations, and a Momentum Preserving Symplectic Integrator, Ph.D. Thesis, UC Berkeley, 1991. 
[22] M.J. Pflaum, Analytic and Geometric Study of Stratified Spaces. Lecture Notes in Mathematics, Volume 510, 2001, Springer-Verlag.

[23] V. Pó́naru, Singularités $C^{\infty}$ en présence de symétrie. En particulier en présence de la symétrie d'un groupe de Lie compact. Lecture Notes in Mathematics, Volume 510, 1976, Springer-Verlag.

[24] M. PutA, Hamiltonian Mechanical Systems and Geometric Quantization, Mathematics and its Applications, Volume 260, Kluwer, 1993.

[25] J.C. Simo, D. Lewis, And J.E. Marsden, The stability of relative equilibria. Part I: The reduced energy-momentum method. Arch. Rat. Mech., 115(1)(1991):15-59.

[26] A. Vanderbauwhede and J.-C. Van Der Meer, A general reduction method for periodic solutions near equilibria in Hamiltonian systems, in Normal Forms and Homoclinic Chaos, W. Langford and W. Nagata, eds., 273-294, Volume 4 of Fields Inst. Commun., Amer. Math. Soc., Providence, RI, 1995.

P. BirTeA

Departamentul de Matematică, Universitatea de Vest, RO-1900 Timişoara, Romania.

Email: birtea@math.uvt.ro

M. PUTA

Departamentul de Matematică, Universitatea de Vest, RO-1900 Timişoara, Romania.

Email: puta@math.uvt.ro

T.S. RATiu

Centre Bernoulli, École Polytechnique Fédérale de Lausanne, CH-1015 Lausanne, Switzerland.

Email: tudor.ratiu@epfl.ch

R. M. TUDORAN

Centre Bernoulli, École Polytechnique Fédérale de Lausanne, CH-1015 Lausanne, Switzerland; Departamentul deMatematică, Universitatea de Vest, RO-1900 Timişoara, Romania.

Email: razvan.tudoran@epfl.ch 